Article

\title{
An Empirical Comparison of Carbon Credit Projects under the Clean Development Mechanism and Verified Carbon Standard
}

\author{
Andrea von Avenarius ${ }^{1, *}$, Thattekere Settygowda Devaraja ${ }^{2}$ and Rüdiger Kiesel ${ }^{1}$ \\ 1 Universität Duisburg-Essen, Universitätsstrasse 12, 45141 Essen, Germany; ruediger.kiesel@uni-due.de \\ 2 Department of Commerce, Post Graduate Centre, Hemaganotri Campus, University of Mysore, \\ Hassan 573220, India; devaraj.uni.mysore@gmail.com \\ * Correspondence: andrea.vonavenarius@uni-due.de
}

Received: 2 May 2018; Accepted: 29 May 2018; Published: 4 June 2018

\begin{abstract}
Carbon credit projects generate carbon credits by abating greenhouse gas emissions. Carbon credits can then be traded on carbon markets or immobilized in order to compensate for caused emissions. The Clean Development Mechanism (CDM) and Verified Carbon Standard (VCS), as the two most important carbon credit mechanisms, are investigated and compared regarding the success of projects. We define success as the fulfilling of the ex-ante emission abatement estimation and apply regression analyses to explain its variation on a project level by technology, location, scale, duration and participation. The results are discussed in detail on technology level for wind power, energy efficiency, hydro power as well as biomass projects and are compared with regard to CDM and VCS. Our main results indicate that large scale projects often compensate for their under-performance due to economies of time. Furthermore, the duration of projects, their location and structure of participants have significant influence on the success of the projects. The sign of the coefficients of explanatory variables are broadly in line with intuition and related literature, although, due to data availability, they are not always highly significant statistically.
\end{abstract}

Keywords: Clean Development Mechanism; Certified Emission Reduction; Verified Carbon Standard; Verified Carbon Unit

\section{Introduction}

Carbon credit trading regimes put a cost-efficient price on carbon emissions and foster investments in clean and low carbon technologies. Carbon credit projects generate carbon credits by abating greenhouse gas (GHG) emissions. For the abatement of one tonne of $\mathrm{CO}_{2}$ equivalent $\left(\mathrm{CO}_{2}\right.$-eq hereafter, is an aggregate measure for GHG, whereby they are expressed with respect to their global warming potential ([1], p. 73)), one carbon credit is issued. Carbon credits can then be traded on the carbon market or be immobilized in order to compensate for caused emissions. This can take place in a mandatory or voluntary framework. There are diverse regimes of mandatory emission trading schemes, such as the Oregon Carbon Dioxide Standard and Regional Greenhouse Gas Initiative in the USA or the European Union Emission Trading Scheme (EU ETS), which is accountable for "over three quarters of international carbon trading" ([2], p. 1). Furthermore, China tested Emission Trading Schemes in several cities and provinces and launched a national ETS in December 2017 ([3], p. 24). According to ([4], p. 23), this would "become the largest carbon pricing initiative in the world, passing the EU ETS". In addition, there are voluntary programs, for instance Gold Standard (GS) or Verified Carbon Standard (VCS) [5]. The different standards have different scopes of emission reduction sometimes in combination with further social engagements as well as differing administrative requirements. Accordingly, the cost and effort to engage in carbon credit projects vary [6]. In some cases, a voluntary 
carbon trading regime is established as a pre-compliance framework before transforming it into a mandatory one [7].

An important carbon credit scheme established in the frame of the Kyoto Protocol is the Clean Development Mechanism (CDM). The CDM is highly regulated, so we refer in the sequel to it as mandatory. Companies from Annex I countries have the opportunity to compensate for emissions in their country of residence with regard to the local emission policies in force by establishing GHG emission reduction projects in developing countries predefined by the Kyoto Protocol. The marginal abatement costs in those developing or least-developed countries are lower compared to those of industrialized countries [8]. For every tonne of $\mathrm{CO}_{2}$-eq avoided, a Certified Emission Reduction (CER) is issued. The CERs can be traded and retired in the EU ETS. However, as the price for emission certificates within the EU ETS fell rapidly in recent years, the value of CERs decreased as well to a low level. Thus, CDM projects became very unattractive for companies and investors and the market came to a standstill. In addition, the carbon market in general is sensitive to economic cycles $[9,10]$. Consequently, the investment in and issuance of carbon credits shrunk past 2008, but recovered within the next years.

According to [11], the CDM Executive Board is evaluating new fields of application, for example the International Civil Aviation Organization's future offset market, in order to revive the CDM market. Moreover, Ref. [12] (p. 10) states that "189 countries representing 96\% of global GHG emissions and $98 \%$ of the world's population have committed to reduce their GHG emissions and adapt to the changing climate through their Intended Nationally Determined Contributions (INDCs)" linked to the United Nations Framework Convention on Climate Change, 21st Conference of the Parties (COP21) in Paris. Accordingly, these countries intend to make efforts towards a low carbon economy for which emission trading is a measure and, thus, rivet on carbon credit projects [12].

The Verified Carbon Standard (VCS) program is the leading voluntary carbon standard by offset issuance, retirement as well as transacted volumes [11]. Verified Carbon Units (VCU) are certificated for each avoided tonne of $\mathrm{CO}_{2}$-eq generated by VCS projects in a voluntary carbon market. These VCS projects face fewer as well as softer administrative barriers and, due to this, lower investment cost [13].

The increasing popularity of climate-neutral products as well as the goal of compensating the business activity's emissions in total lead companies to engage in carbon abatement projects and actively participate in carbon markets. Refs. [13,14] identify mainly Corporate Social Responsibility as motivation for the engagement in the voluntary carbon markets.

We will identify the driving factors of successful CDM and VCS projects, respectively, as representatives for mandatory and voluntary carbon credit regimes. In order to do so, we will analyze the types of carbon emission reduction projects separately and compare our findings. The performance of a project can be measured by reaching the ex-ante estimated quantity of carbon credits generated by the specific project. Identifying the determinants of successful CDM and VCS projects is relevant due to the existing difficulties within these markets as market prices of CERs as well as VCUs having declined heavily together with the number of projects.

The comparison of compliance and voluntary carbon offset success drivers is of interest for companies as well as project developers who invest in emission reduction projects. Knowledge of success drivers allows for adjusting to difficulties and avoiding losses. In addition, our results are of interest to policy makers who design the corresponding carbon markets and set investment incentives. The attractiveness and lucrativeness of carbon markets can be increased. Furthermore, the carbon emission abatement and reduction will be more effective and efficient. Especially, since the number and size of ETS have risen considerably, the determinants of carbon projects generating tradable certificates are of major concern (see [12]).

A related work ([15], p. 3) concentrates on "the ratio between actual CERs issued and estimated" in order to identify determinants on the CDM project success and to adjust procurement strategies with regard to the CER production satisfying the Kyoto Protocol and the EU ETS commitments. They investigate CERs with regard to host country, unilateral/bilateral project setup, type of project 
developer, project category, type and size, designated operational entity in charge of validation, the quality of the additionality argumentation, the quality of the stakeholder consultation as well as the quality of the expected sustainability benefits as stated in the project design documents. Additionally, they conduct interviews with CDM experts, such as project developers. Their results indicate that many CDM projects are under-performing and bilateral projects are more successful than unilateral, especially when European project partners are involved due to "improved access to technology, technical support, quality control and upfront financing" ([15], p. 6). They claim that renewable energy and energy efficiency projects perform properly due to their monitoring methodologies, which are not as complex as for the poorly performing waste projects. Hydro power projects have a rate of issuance of $93 \%$ and, thus, they perform best. Moreover, small CDM projects, in particular issuing below 20,000 CERs per year, and very large projects with more than 540,000 CERs per year are successful.

Refs. $[6,16]$ focus on explaining the prices of the primary CDM market and voluntary market, respectively. On the primary market, the initial transaction takes place and the project developer sells the offset. Intermediaries trade carbon credits on the secondary market [10]. Ref. [6] estimates hedonic price functions considering diverse determinants, such as technology, host nation's stage of development and region among others. They find offset prices to be higher, if the host nation is a developing or least-developed country. In addition, they find price premia for carbon credits that are CDM- or GS certified, whereas VCUs face a price discount. In contrast, Ref. [16] applies an asset pricing approach and finds that the "primary market is characterised by the preemptive behaviour of carbon firms, and, potentially, speculation and inefficiencies of information transmission mechanism between secondary and the primary market segments" (p. 88).

Focusing on nation level characteristics as explanatory variables, Ref. [17] applies cluster analysis to potential CDM host countries in order to identify their attractiveness with regard to mitigation potential, institutional CDM capacity and general investment climate. She finds the regions of Latin America and Asia to host the most promising CDM countries while African CDM host countries signify rather low attractiveness. Ref. [18] conducts regression analyses in order to explain the differential distribution of CDM projects across host countries. They find human capital, GHG emission levels, amount of CERs and countries offering a growing market for carbon credit project by-products to be fostering the CDM activity of a host country.

Ref. [19] analyzed CERs only. They examined the cost structure of CERs with regard to different project technologies, location, scale and time. Methodically, they apply ordinary least square (OLS) estimation [19]. The main findings are: economies of scale exist in the cost of CERs; the effects of scale vary across technologies; average CER costs decline over project duration; projects with a later start compared to others have higher average cost in CERs; the distribution of CDM projects regarding location or technology does not ensue the relative cost structure and the cost of CERs varies significantly depending on the project location.

All mentioned studies focus on CER only and do not systematically compare CDM and VCS performance. In contrast, our study sheds light on similarities and differences in success factors for CDM and VCS projects, which has not been done before. We explain the rate of carbon credit issuance considering the projects' technology, methodology, location, duration and uni-/multilateral specifications.

This paper is organized as follows: the second section explains the conceptual model and the data used. Section 2 analyzes the performance of CDM and VCS projects. Section 3 discusses the results. Finally, Section 4 summarizes and concludes this paper.

\section{Analyzing Factors Driving Successful Carbon Projects}

The aim of our analysis is to find the determinants of successful carbon credits generating projects with respect to mandatory and voluntary carbon regimes. As CDM projects have so far generated most of the carbon credits worldwide [11], we focus on CDM projects representative for the mandatory framework. Germany is among the most dedicated Annex I countries regarding its engagement in 
CDM activities [20]. The VCS program has the largest market share in the voluntary carbon market [11]. About 18\% of the VCUs issued from 2012 until November 2016 originated from India and India hosts about $30 \%$ of all VCS projects. By that, India is the leading VCS host country. We choose all VCS projects hosted in India (VCS projects hereafter) as data representative of the voluntary carbon market.

\subsection{Methodology}

For the purpose of identifying the relevant factors of the success of carbon credit projects, we first apply Ordinary Least-Squares (OLS) regressions to the separate carbon project groups. According to the Gauss-Markov theorem, the OLS estimator does not necessarily need the regression residuals to be normally distributed in order to yield a best linear unbiased estimator (blue) ([21], pp. 102-105). Secondly, we employ stepwise regressions and allow the predictor variables to interact. Here, only a subset of predictor variables, and combinations of those, is selected by adding and removing from a linear model with respect to their statistical significance in explaining the response variable (for detailed explanation and discussion, see [22,23], pp. 92-98, 364-369). We define the logarithmized quotient of actual and estimated emission reductions, $\log (\mathrm{q})$, as success indicator and response variable as inspired by [15]. If this quotient is close to 0 , a project is considered successful. A project with a quotient larger than 0 would, consequently, be over-performing. We regress the log quotients on the candidate variables. The candidate variables are project-specific information-for example, the project's technology. CDM projects with German involvement cover the technologies wind and hydro power, energy efficiency, biomass, gas to power and others. Gas to power projects cover biogas, landfill gas and coal bed/mine methane to energy projects. CDM projects of the categories geothermal, transport, cement, catalytic N2O reduction and HFC23 decomposition are subsumed by "others". The VCS projects cover the technologies biomass, energy efficiency, wind and hydro power, and others. The category "others" comprises projects of the categories traffic, reforestation, solar power, $\mathrm{N}_{2} \mathrm{O}$ reduction and Perfluorocarbon gases abatement, chemical recovery and composting. Energy efficiency projects concerning both CDM and VCS cover fuel switching and waste heat recovery projects, district heating as well as energy efficiency measures concerning households, industry, own power generation or the energy supply side. Dummy variables represent the projects' technology; here, the technology category wind power serves as base. The different project technologies imply different levels of complexity with regard to the planning, administration and execution of the project activity.

The project's methodology is defined by the United Nations Framework Convention on Climate Change (UNFCCC) and distinguishes between large and small scale projects [24]. According to those methodologies, the emission reductions are calculated and documented. We also categorize the VCS projects according to these definitions. The projects' scale is transferred into a dummy variable, where the small scale specification serves as base. This differentiation allows for addressing a question similar to $[19,25]$ related to the projects' cost perspective concerning the existence of economies of scale for the certificate issuance. Furthermore, the number of countries involved in a project are candidate variables. Here, we want to investigate if it is advantageous to invest in a bilateral project or if there are significant economies of scale when there are diverse countries engaged. The logarithmized actual crediting time, $\log (\mathrm{t})$, describing the time span for which carbon credits have been issued, also serves as a success factor in order to identify economies of time. In addition, we take into account the project's region of implementation for the CDM projects with German participation. This is of interest due to the influence of the general investment climate in the designated regions on the project activity, which might be affected by political unrest, infrastructure and human development among others $[17,18]$. We distinguish between Asia, Africa and Latin America following [19]. In addition, these regional variables are dummy variables and Asia as the host region serves as base. As we focus our VCS analysis on India as host country, we do not take into account further regional variables. 


\subsection{Data}

CDM projects are well documented by regulation on the UNFCCC websites (http:/ /cdm.unfccc. int/), which provide standardized data concerning the projects. All CDM projects undergo a specific administrative cycle consisting of seven steps [26]. First, the project design document is prepared using the approved emission baselines and monitoring methodology. Here, the project is presented with regard to the applied technology, location, size, scale and project participants. In a second step, the Designated National Authority of the party engaged in the project must confirm that the country has ratified the Kyoto Protocol, that participation is not compulsory and that the host country attests the project's contribution to sustainable development. Then, the project design document is validated by a third-party certifier, such as TUEV NORD CERT GmbH (Essen, Germany) (https: //www.tuev-nord.de/en/company/certification/), and the project is registered by the CDM Executive Board. In a fifth step, the project participant needs to submit monitoring reports documenting the project activity and the actual emission abatement. Finally, the monitoring reports are verified by the third-party certifier and the CERs are issued by the Executive Board. Equivalent to that, the VCS projects are documented at the Verified Carbon Standard website (http:/ / www.v-c-s.org/). During the administrative process, in order to conduct a carbon credit project, the project developers must, among other things, estimate the yearly emission abatement in $\mathrm{CO}_{2}$-eq. This is done in the project design document, where further technical and administrative information is also given. Moreover, within the projects' lifetime monitoring reports, which document the project's emission abatement, need to be handed in to the responsible carbon standard. After reviewing the monitoring report, the carbon credits are issued. Thus, the estimated and actual amount of emission abatement is supervised and transparent.

We investigate 200 CDM projects with German participation and 303 VCS projects hosted in India, which have been issuing CERs and VCUs, respectively, up to the end of 2014.

The logarithmized quotient of actual and estimated emission reductions as success indicator is shown in Figure 1. On average, the carbon credit projects under perform with means of -0.32 and -0.21 for German CDM projects and Indian VCS projects, respectively. The samples' variances are 0.27 for German CDM projects and 0.17 for VCS projects. Although the CDM projects are said to be more regulated compared to voluntary carbon abatement projects, which might imply increased technical and administrative quality control, the German CDM projects show the highest variance combined with the lowest mean of project success. One explanation for the under-performance of the carbon credit projects is the presence of ramp-up phases during which the estimated annually emission reductions are not yet met. Unfortunately, those phases are not evident in the project documentation and, consequently, the corresponding projects were not excluded from the sample.

Figure 2 gives an overview of the carbon credit projects under observation regarding their scale and technology. The number of small scale projects is indicated by black color. The top graph displays the number of CDM projects with German participation by technology and scale. About $57 \%$ of the CDM projects with German participation are large scale projects. Moreover, wind power together with hydro power projects cover roughly $50 \%$ of the German CDM projects under consideration. The lower graph shows the VCS projects as well by technology and scale. Wind power projects are, here, by far the dominant technology with $68 \%$ followed by biomass with about $14 \%$. The majority of VCS projects considered here are small scale projects $(60 \%)$.

In addition, $81.5 \%$ of those CDM projects in the sample are hosted in Asia, $6 \%$ and $12.5 \%$ in Africa and Latin America, respectively. Associated with this are the findings of [19], in which fixed project costs are significantly higher in the regions of Africa, Latin America and Oceania compared to Asia. Among others, Ref. [27] explains the distribution of CDM projects by avoidance of investments in regions of political unrest and that national GHG emissions, gross savings, population and GDP enhance the investment climate. Ref. [18] explains that human capital, GHG emission levels as well as emerging markets for CDM by-products influence the allocation of CDM projects. Concentrating on Indian renewable energy CDM projects, [28] identify state level policies as a significant influence on 
the distribution of projects across India and that existing renewable energy capacity attracts larger renewable energy CDM power projects.
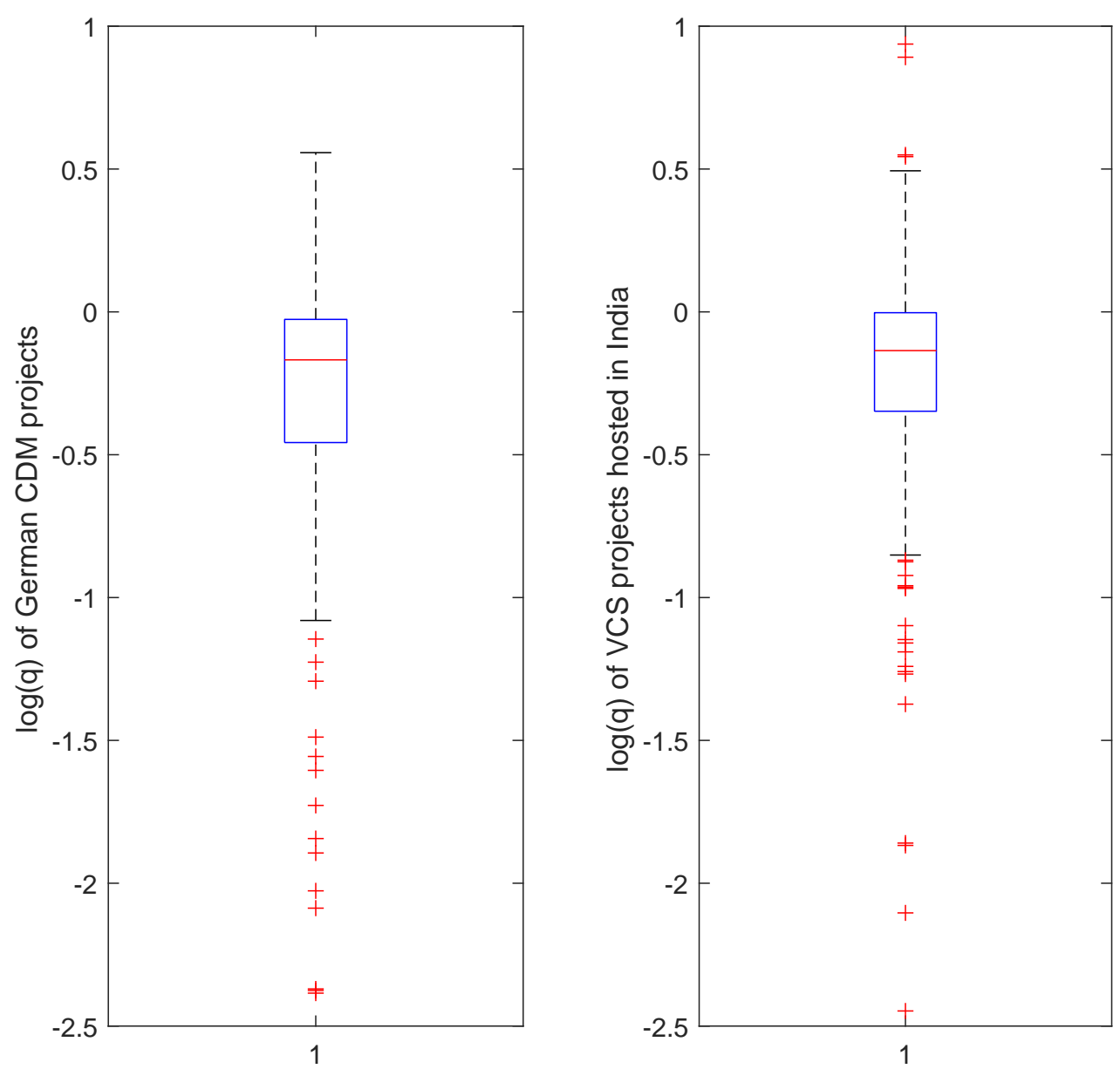

Figure 1. Boxplots of logarithmized quotients of actual and estimated emission reductions of CDM projects with German participation (left) and VCS projects hosted in India (right).

On average, four countries are involved in a CDM project with German participation. In contrast, the average number of countries involved in a VCS project is 1.08 . Only $6.6 \%$ of the VCS projects under consideration have an international project partner.

In addition, the average duration of a carbon credit project is calculated as the time span where CER and VCU issuance occurred, respectively. On average, the issuance period regarding CDM projects was 3.8 years and 3.5 years with respect to VCS projects. We consider CDM and VCS data from 2000 until 2014. 

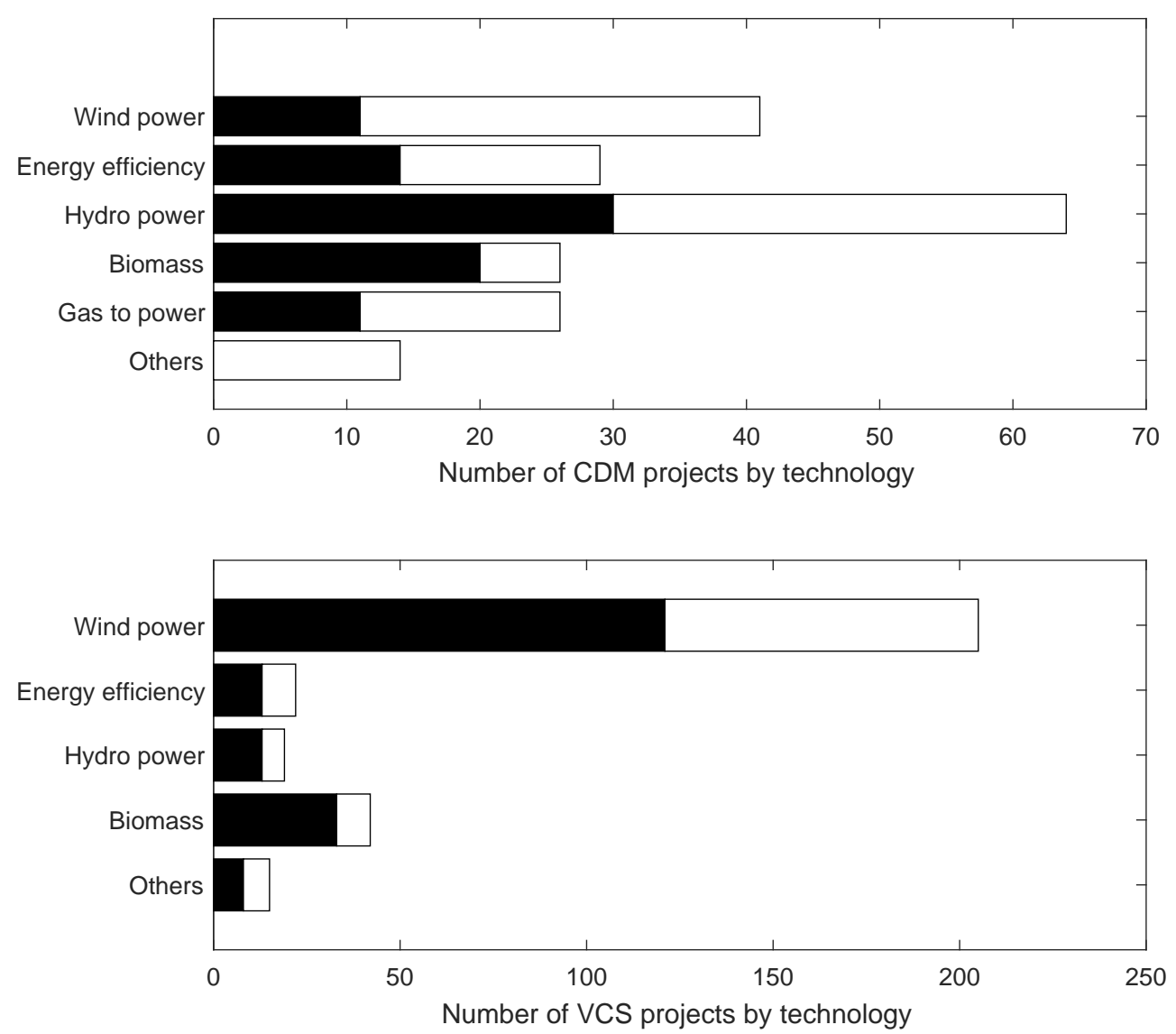

Figure 2. CDM projects with German participation (top) and VCS projects hosted in India (bottom) by technology and scale (small scale is indicated by black color, large scale is indicated by white color).

\section{Empirical Findings}

In Table 1, we list the explanatory variables with influence on the success of carbon credit projects for each project group and their corresponding OLS coefficient estimates.

Table 1. OLS regression results for German CDM projects and VCS projects hosted in India; ** denotes significance at the $5 \%$ level

\begin{tabular}{ccc}
\hline & CDM & VCS \\
\hline Constant & $-0.2983^{* *}$ & 0.0029 \\
Energy efficiency & -0.0826 & -0.0233 \\
Hydro power & -0.0735 & $0.2329 * *$ \\
Biomass & -0.1024 & 0.0246 \\
Gas to power & -0.1019 & \\
Others & 0.0274 & -0.0415 \\
Latin America & -0.0680 & \\
Africa & -0.2269 & \\
Scale & 0.0257 & -0.0762 \\
log(t) & 0.0967 & 0.0052 \\
Countries & -0.0152 & $-0.1911^{* *}$ \\
$R$-squared & 0.0509 & 0.0565 \\
Observations & 200 & 303 \\
\hline
\end{tabular}

The explanatory power of these regressions is limited with respect to the coefficient of determination, $R^{2}$, which is a common goodness of fit measure with regard to regressions. 
The OLS regression identifies no significant influence on the success of German CDM projects. Nevertheless, compared to the listed technologies, CDM wind power projects perform best, with the technology category "others" being an exception. Regarding the VCS projects' success, hydro power projects perform significantly better than wind power projects at the $5 \%$ level of significance. Additionally, VCS projects with many countries involved are less successful with significance at the $5 \%$ threshold. The number of countries influence on the CDM projects is also negative yet not statistically significant. Moreover, the projects' duration has a positive, but not significant, influence on the projects' success. This holds for CDM and VCS projects. The projects' scale influences the rate of certificate issuance positively with regard to CDM projects and negatively with respect to VCS projects. Again, the coefficients are statistically not significant. Further regression results with interactions of the coefficients can be requested from the authors. In the remaining work, we will examine the carbon credit projects on technology level.

\subsection{Wind Power Projects}

Focusing on wind power projects, the samples' means regarding $\log (\mathrm{q})$ are -0.22 and similar for CDM and VCS projects. The variance for CDM wind power projects is 0.09 while the one for VCS wind power projects is 0.13 . Here, the CDM sample's mean is larger and variance smaller than the average. In addition, the VCS projects' mean is equal to the corresponding CDM mean, but smaller than the overall VCS mean; nevertheless, the variance is smaller compared to the sample variance. We find little explanatory power with regard to the OLS regression of VCS projects. In contrast, we can explain about $18 \%$ of the variation in $\log (\mathrm{q})$ with the help of the factors listed in Table 2 concerning CDM wind power projects. In both cases, projects with a longer credit issuing time are more successful compared to shorter durations at a significance level of 5\% and $1 \%$ for CDM and VCS projects, respectively. This hints at learning effects. As found in the general OLS regression results for CDM and VCS projects, large scale CDM projects are more successful compared to small scale CDM projects, whereas the opposite holds for VCS projects. However, the corresponding coefficients are not statistically significant.

Table 2. OLS regression results for CDM and VCS wind power projects; ${ }^{* *}$ and ${ }^{* * *}$ denotes significance at the $5 \%$ and $1 \%$ level, respectively.

\begin{tabular}{ccc}
\hline & CDM & VCS \\
\hline Constant & $-0.5215^{* * *}$ & $-0.3093^{* * *}$ \\
Latin America & 0.1272 & \\
Africa & -0.0991 & \\
Scale & 0.2013 & -0.0592 \\
$\log (\mathrm{t})$ & $0.2580^{* *}$ & $0.1183^{* * *}$ \\
Countries & -0.0500 & 0.0077 \\
R-squared & 0.1780 & 0.0539 \\
Observations & 41 & 205 \\
\hline
\end{tabular}

Applying stepwise regression, the coefficients of determination are $23 \%$ and $6 \%$ for CDM and VCS wind power projects, respectively. Table 3 displays the stepwise regression factors and coefficients for $\mathrm{CDM}$ wind power projects. Accordingly, an increased number of participating countries reduces the projects' success with significance at the $5 \%$ threshold indicating, for instance, communicative or administrative difficulties. The projects' duration and scale have no significant singular influence on the projects' success. The corresponding coefficients indicate that large scale CDM wind power projects perform worse than small scale projects and that there are learning effects during the conduct of CDM wind power projects. However, large scale projects with increasing durations outperform small scale projects with significance at the $10 \%$ threshold and suggest that the disadvantages linked to the large scale project specification is overcome by time. This implies economies of scale and time. 
Still, balancing effects might be present. According to the stepwise regression results, the projects' location does not determine the success of CDM wind power projects.

Table 3. Stepwise regression results for CDM wind power projects; $R^{2}=0.2301 ;{ }^{*}$ and ${ }^{* *}$ denotes significance at the $10 \%$ and $5 \%$ level, respectively.

\begin{tabular}{ccccc}
\hline & Estimate & SE & $t$ Stat & $p$-Value \\
\hline Constant & -0.1325 & 0.2842 & -0.4661 & 0.6439 \\
Scale & -0.2705 & 0.2982 & -0.9071 & 0.3704 \\
$\log (\mathrm{t})$ & 0.0546 & 0.1520 & 0.3593 & 0.7215 \\
Countries & $-0.0615^{* *}$ & 0.0287 & -2.1428 & 0.0390 \\
Scale $\times \log (\mathrm{t})$ & $0.3366^{*}$ & 0.1979 & 1.7006 & 0.0976 \\
\hline
\end{tabular}

Concerning VCS wind power projects, Table 4 shows the stepwise regression results. Accordingly, we are able to explain about $7 \%$ of $\log (\mathrm{q})$ 's variation. The number of participating countries does not influence the VCS wind power projects' success significantly and is omitted by the stepwise regression algorithm. Large scale VCS wind power projects perform worse than small scale ones at the $5 \%$ threshold. The projects' duration has positive, yet statistically insignificant, influence on the rate of VCU issuance. Large scale VCS wind power projects with increased projects durations have an increased rate of VCU issuance at the $10 \%$ level of significance indicating economies of scale and time.

Table 4. Stepwise regression results for VCS wind power projects; $R^{2}=0.0666 ;{ }^{*}, * *$ and ${ }^{* * *}$ denotes significance at the $10 \%, 5 \%$ and $1 \%$ level, respectively.

\begin{tabular}{ccccc}
\hline & Estimate & SE & $t$ Stat & $p$-Value \\
\hline Constant & $-0.2271^{* * *}$ & 0.0676 & -3.3601 & 0.0009 \\
Scale & $-0.1748^{* *}$ & 0.0865 & -2.0215 & 0.0446 \\
$\log (\mathrm{t})$ & 0.0415 & 0.0615 & 0.6740 & 0.5011 \\
Scale $\times \log (\mathrm{t})$ & $0.1369^{*}$ & 0.0825 & 1.6587 & 0.0987 \\
\hline
\end{tabular}

\subsection{Energy Efficiency Projects}

The mean and variance of CDM and VCS energy efficiency projects are -0.4 and -0.31 as well as 0.33 and 0.24 , respectively. Table 5 shows the OLS regression results for CDM and VCS energy efficiency projects. The variation of $\log (\mathrm{q})$ can be explained by $26 \%$ and $13 \%$ concerning CDM and VCS projects, respectively. Focusing on CDM projects, only the coefficient of the host region Africa is significant at the $10 \%$ level. This suggests that Africa as host region is inferior to the host region Asia with regard to the rate of CER issuance. None of the coefficients regarding the VCS projects are significant. However, Table 5 indicate economies of scale and time as the scale's and project's durations coefficients are positive.

Table 5. OLS regression results for CDM and VCS energy efficiency projects; ${ }^{*}$ denotes significance at the $10 \%$ level.

\begin{tabular}{ccc}
\hline & CDM & VCS \\
\hline Constant & -0.4185 & -0.8099 \\
Latin America & -0.3767 & \\
Africa & $-0.5847^{*}$ & \\
Scale & 0.2791 & 0.0943 \\
$\log (\mathrm{t})$ & 0.0483 & 0.2336 \\
Countries & -0.0067 & 0.2642 \\
$R$-squared & 0.2250 & 0.1317 \\
Observations & 29 & 22 \\
\hline
\end{tabular}


The stepwise regression results for CDM energy efficiency projects are displayed in Table 6 . Accordingly, the projects' scale has no significant influence on the projects' success and is omitted by the stepwise regression algorithm, whereas the host region Africa is disadvantageous compared to Asia with significance at the $5 \%$ level. This confirms our result in Table 5. The African CDM energy efficiency host countries, namely Nigeria, Lesotho, Rwanda and Zambia, are considered to be "very unattractive" CDM host countries ([17], p. 2183). In contrast, energy efficiency projects located in Latin America are more successful compared to those in Asia, yet the corresponding coefficient is not statistically significant. Moreover, projects located in Latin America have an increased rate of CER issuance when the project duration increases with significance at the $10 \%$ threshold. Nevertheless, an increasing number of participating countries in energy efficiency projects hosted in Latin America reduce the projects' success with significance at the $10 \%$ level. Energy efficiency CDM project host countries in Latin America are Argentina, Bolivia and Brazil. Argentina and Brazil are characterized as "very attractive" CDM host countries, whereas Bolivia is identified to be "attractive to a limited extent" ([17], p. 2183). Asian host countries for the CDM energy efficiency projects under consideration are China, Bangladesh, India, Indonesia and Pakistan. Thereof, China, India and Indonesia are "very attractive", Bangladesh is "attractive to a limited extent" and Pakistan is considered "very unattractive" as CDM host country by [17], p. 2183. The number of countries involved in CDM energy efficiency projects also has negative, but not statistically significant, singular influence on the success of CDM energy efficiency projects with respect to OLS and stepwise regression. The projects' duration also has negative, but statistically insignificant influence on the success of CDM energy efficiency projects according to our stepwise regression results.

Stepwise regression identifies no significant factor determining the success of VCS energy efficiency projects.

Table 6. Stepwise regression results for CDM energy efficiency projects; $R^{2}=0.4102 ;{ }^{*}$ and ${ }^{* *}$ denotes significance at the $10 \%$ and $5 \%$ level, respectively.

\begin{tabular}{ccccc}
\hline & Estimate & SE & $t$ Stat & $p$-Value \\
\hline Constant & -0.1998 & 0.1557 & -1.2839 & 0.2125 \\
Latin America & 1.0631 & 1.9092 & 0.5568 & 0.5833 \\
Africa & $-0.7333^{* *}$ & 0.2753 & -2.6642 & 0.0142 \\
$\log (\mathrm{t})$ & -0.0274 & 0.1290 & -0.2125 & 0.8337 \\
Countries & -0.0130 & 0.0227 & -0.5723 & 0.5730 \\
Latin America $\times \log (\mathrm{t})$ & $0.9721^{*}$ & 0.5319 & 1.8277 & 0.0812 \\
Latin America $\times$ Countries & $-1.0048^{*}$ & 0.5834 & -1.7222 & 0.0991 \\
\hline
\end{tabular}

\subsection{Hydro Power Projects}

CDM hydro power projects are slightly less successful than CDM projects in general with a mean of $\log (\mathrm{q})$ of -0.33 . The variance of the projects' success is 0.34 . Furthermore, VCS hydro power projects are the most successful within this work with an average $\log (\mathrm{q})$ of 0.03 and a corresponding variance of 0.12 , which is interesting from an investor's point of view. All VCS hydro power projects have only the host country as participating country. The variation of the success indicator can be explained by $33 \%$ and $25 \%$ regarding CDM and VCS projects with the help of OLS regression, respectively (see Table 7). Here, CDM hydro power projects located in Latin America are significantly more successful than those in Asia at the 1\% threshold. Host countries of CDM hydro power projects in Latin America are Ecuador, Guatemala, Honduras, Peru and Costa Rica. The latter is characterized to be an attractive CDM host country, whereas, in [17], p. 2183, the other countries are considered as being "attractive to a limited extent". However, the CDM hydro power host countries in Asia are China, India, Laos, Vietnam and Pakistan. China and India are categorized as "very attractive", Laos and Vietnam as "attractive to a limited extent" and Pakistan as "very unattractive" as CDM host country ([17], p. 2183). Again, among others, singular political events, environmental issues or structural differences within 
a country might be the reason for this contradictory result, as the influential factors are not evident on a country level, as discussed by [17]. Moreover, an increasing number of participating countries reduces the success of CDM hydro power projects significantly at the $1 \%$ level, which is hinting at communicative discrepancies or increased administrative difficulties delaying the issuance of CERs.

Table 7. OLS regression results for CDM and VCS hydro power projects; ** and ${ }^{* * *}$ denotes significance at the $5 \%$ and $1 \%$ level, respectively.

\begin{tabular}{ccc}
\hline & CDM & VCS \\
\hline Constant & -0.1365 & 0.1475 \\
Latin America & $0.8825^{* * *}$ & \\
Africa & 0.2433 & \\
Scale & 0.0412 & 0.1778 \\
$\log (\mathrm{t})$ & 0.0845 & $-0.2116^{* *}$ \\
Countries & $-0.1126^{* * *}$ & \\
$R$-squared & 0.3259 & 0.2451 \\
Observations & 64 & 19 \\
\hline
\end{tabular}

Concerning VCS hydro power projects, the projects' duration influences the rate of VCU issuance negatively with significance at the $5 \%$ threshold. This implies the absence of learning effects in the projects' conduct.

In addition, $63 \%$ of the variation in $\log (q)$ concerning CDM hydro power projects can be explained by stepwise regression. Table 8 states the explanatory variables and the estimated coefficients. Accordingly, the projects location Latin America and Africa are beneficial compared to Asia each with significance at the $1 \%$ level. However, there are balancing effects, as large scale projects in Latin America have a decreased rate of CER issuance with significance at the 5\% level. CDM hydro power projects hosted in Africa with an increased number of participating countries also have a reduced rate of CER issuance significant at the 1\% threshold. Namely, the relevant countries are Kenya and Uganda. Kenya is identified to have "rather low institutional CDM capacity, low mitigation potential and a relatively bad investment climate" ([17], p. 2177). Uganda is identified to be of medium to very high CDM capacity, low mitigation potential and bad investment climate and "attractive to a limited extent" for CDM ([17], p. 2183). Large scale projects perform significantly worse than small scale projects at the $1 \%$ level. The projects' duration has significant negative influence on their success at the $10 \%$ threshold. However, the disadvantages of the large scale project specification are overcome by time with significance at the $1 \%$ threshold implying economies of scale and time. Additionally, the number of participating countries shows a significantly negative influence on the rate of CER issuance at the $1 \%$ threshold. This indicates administrative or communicative difficulties leading to delays in CER issuance. These difficulties are overcome by time with significance at the $1 \%$ level. Furthermore, large scale CDM hydro power projects with an increasing number of participating countries have an increased rate of carbon credit issuance significant at the $5 \%$ level.

Furthermore, $19 \%$ of the variation of $\log (q)$ can be explained by the stepwise regression results shown in Table 9. Here, only the duration of the projects is identified to have significant negative influence on the projects' success at the $10 \%$ level. This indicates that no learning effects are present equivalent to the result in Table 7. 
Table 8. Stepwise regression results for CDM hydro power projects; $R^{2}=0.6255 ;{ }^{*}{ }^{* *}$ and ${ }^{* * *}$ denotes significance at the $10 \%, 5 \%$ and $1 \%$ level, respectively.

\begin{tabular}{ccccc}
\hline & Estimate & SE & $t$ Stat & $p$-Value \\
\hline Constant & $0.4113^{* *}$ & 0.2005 & 2.0513 & 0.0452 \\
Latin America & $0.8283^{* * *}$ & 0.2559 & 3.2368 & 0.0021 \\
Africa & $11.2064^{* * *}$ & 1.9449 & 5.7619 & 0.0000 \\
Scale & $-0.7938^{* * *}$ & 0.2321 & -3.4196 & 0.0012 \\
$\log (\mathrm{t})$ & $-0.2775^{*}$ & 0.1555 & -1.7842 & 0.0801 \\
Countries & $-0.2848^{* * *}$ & 0.0559 & -5.0933 & 0.0000 \\
Latin America $\times$ Scale & $-1.1202^{* *}$ & 0.4326 & -2.5895 & 0.0124 \\
Africa $\times$ Countries & $-1.2405^{* * *}$ & 0.2225 & -5.5763 & 0.0000 \\
Scale $\times \log (\mathrm{t})$ & $0.5357^{* * *}$ & 0.1849 & 2.8965 & 0.0055 \\
Scale $\times$ Countries & $0.1191^{* *}$ & 0.0458 & 2.5998 & 0.0121 \\
$\log (\mathrm{t}) \times$ Countries & $0.1073^{* * *}$ & 0.0322 & 3.3316 & 0.0016 \\
\hline
\end{tabular}

Table 9. Stepwise regression results for VCS hydro power projects; $R^{2}=0.1876$; ${ }^{*}$ denotes significance at the $10 \%$ level.

\begin{tabular}{ccccc}
\hline & Estimate & SE & $\boldsymbol{t}$ Stat & $\boldsymbol{p}$-Value \\
\hline Constant & 0.1793 & 0.1063 & 1.6862 & 0.1100 \\
$\log (\mathrm{t})$ & $-0.1827^{*}$ & 0.0922 & -1.9814 & 0.0640 \\
\hline
\end{tabular}

\subsection{Biomass Projects}

Compared to the overall mean of CDM projects, CDM biomass projects perform almost equally with a mean of -0.31 regarding the logarithmized quotient of actual and estimated emission reductions. However, the associated variance is higher than the average with 0.28 . The VCS biomass projects are under-performing, yet slightly less than the corresponding CDM projects, with a mean of -0.19 and a variance of 0.22 . Accordingly, the biomass projects' success is rather variable.

All CDM biomass projects are either located in Asia or Latin America. Thus, the host region Africa is omitted as an explanatory variable here. Table 10 shows the regression coefficients for both CDM and VCS projects' regressions. We are able to explain about 33\% of the variation in $\log (q)$ with the help of those coefficients with regard to CDM projects. According to Table 10, there are economies of time concerning CDM biomass projects as the rate of CER issuance increases over time with significance at the $5 \%$ level. With regard to VCS biomass projects, we are able to explain about $10 \%$ of $\log (\mathrm{q})^{\prime} \mathrm{s}$ variation. None of the coefficients are statistically significant. However, the large scale specification influences the VCS biomass projects' success negatively. The same holds for the projects' duration. Consequently, the findings are opposite to the results for CDM biomass projects. Only the number of involved countries increases the rate of VCU issuance.

Table 10. OLS regression results for CDM and VCS biomass projects; ${ }^{* *}$ and ${ }^{* * *}$ denotes significance at the $5 \%$ and $1 \%$ level, respectively.

\begin{tabular}{ccc}
\hline & CDM & VCS \\
\hline Constant & $-1.2294^{* * *}$ & -0.3317 \\
Latin America & 0.4804 & \\
Scale & 0.4056 & -0.1916 \\
$\log (\mathrm{t})$ & $0.6731^{* *}$ & -0.1759 \\
Countries & -0.0831 & 0.2716 \\
$R$-squared & 0.3328 & 0.1047 \\
Observations & 26 & 42 \\
\hline
\end{tabular}


With the help of stepwise regression, we can increase the percentage of explained variation in $\log (\mathrm{q})$ in the case of CDM biomass projects up to $73 \%$ according to Table 11 . Consequently, long project durations decrease the rate of CER issuance with significance at the $1 \%$ level indicating the absence of learning effects. In addition, an increasing number of participating countries significantly reduces the project's success at the $1 \%$ level. This is opposite to our finding in Table 10, where the projects' duration has a positive influence on their success. However, the project duration combined with the number of participating countries has a significantly positive influence on the rate of CER issuance at the $1 \%$ level. This somehow sets off the singular influence of $\log (\mathrm{t})$. Thus, learning effects are present when diverse countries are involved and are able to bring in their experience as well as overcome administrative or communicative difficulties within a reasonable time span. The projects' scale has no significant singular influence on the success of CDM biomass projects; however, the coefficient is negative and indicates that large scale projects under perform. The combination of the large scale specification and the host region Latin America has a significant positive coefficient at the $1 \%$ threshold. This implies that large scale CDM biomass projects in Latin America perform best. The CDM biomass host countries in Latin America are Costa Rica and Brazil. Costa Rica is characterized as "attractive for CDM" ([17], p. 2178). Brazil is identified to have "an exceptionally high mitigation potential, a good institutional CDM capacity and a good investment climate" ([17], p. 2178). Nevertheless, 22 of the 26 CDM biomass projects are hosted in Asia, whereof 16 are located in India, while the others are hosted in China, Malaysia and Indonesia. China, India and Indonesia are identified to be among the "CDM (non-sink) stars" according to ([17], p. 2182), whereas Malaysia is characterized as "attractive" CDM host country ([17], p. 2183). Furthermore, large scale CDM biomass projects with many countries involved have an increased rate of CER issuance with significance at the $5 \%$ level.

In contrast to CDM biomass projects the projects' scale has no influence on the success of VCS biomass projects with reference to the stepwise regression results in Table 12 and is omitted as an explanatory variable. Moreover, the rate of VCU issuance decreases with an increase of credit issuing time significantly at the $5 \%$ threshold. This means that the rate of issuance shows a negative development over time and hints at problems in the conduct of the project activities as no learning effects seem to be present. Additionally, the number of participating countries reduces the projects' success significantly at the $5 \%$ level. This is set off by the factor combination of $\log (\mathrm{t})$ and the number of involved countries, as this combination has a significant positive influence on the projects' success at the $5 \%$ threshold. Thus, there are learning effects the longer the projects continue and the more countries are involved. With the factors and coefficients identified in Table 12, we can explain 17\% of the variation in $\log (q)$.

Table 11. Stepwise regression results for international CDM biomass projects; $R^{2}=0.7255 ;{ }^{* *}$ and ${ }^{* * *}$ denotes significance at the $5 \%$ and $1 \%$ level, respectively.

\begin{tabular}{ccccc}
\hline & Estimate & SE & $t$ Stat & $p$-Value \\
\hline Constant & $3.9996^{* * *}$ & 1.2228 & 3.2709 & 0.0042 \\
Latin America & 0.1464 & 0.2309 & 0.6338 & 0.5342 \\
Scale & -0.4399 & 0.4039 & -1.0889 & 0.2905 \\
$\log (\mathrm{t})$ & $-1.9406^{* * *}$ & 0.6105 & -3.1788 & 0.0052 \\
Countries & $-2.0112^{* * *}$ & 0.4352 & -4.6208 & 0.0002 \\
Latin America $\times$ Scale & $2.8768^{* * *}$ & 0.6426 & 4.4769 & 0.0003 \\
Scale $\times$ Countries & $0.1987^{* *}$ & 0.0933 & 2.1287 & 0.0473 \\
$\log (\mathrm{t}) \times$ Countries & $0.9593^{* * *}$ & 0.2119 & 4.5275 & 0.0003 \\
\hline
\end{tabular}


Table 12. Stepwise regression results for VCS biomass projects; $R^{2}=0.1690$; ${ }^{* *}$ denotes significance at the $5 \%$ level.

\begin{tabular}{ccccc}
\hline & Estimate & SE & $t$ Stat & $p$-Value \\
\hline Constant & 0.1978 & 0.3982 & 0.4968 & 0.6222 \\
$\log (\mathrm{t})$ & $-0.9326^{* *}$ & 0.3825 & -2.4378 & 0.0196 \\
Countries & -0.2513 & 0.3602 & -0.6978 & 0.4895 \\
$\log (\mathrm{t}) \times$ Countries & $0.6811^{* *}$ & 0.3307 & 2.0597 & 0.0463 \\
\hline
\end{tabular}

\subsection{Discussion}

Mandatory, in contrast to voluntary, carbon regimes potentially change the cost structure of obliged companies permanently, depending on carbon prices as carbon credits can be seen as a factor of production as discussed by [29,30]. Our results suggest, in general, that CDM wind power projects are not outperformed by energy efficiency, hydro power, biomass or gas to power projects (see Table 1). In contrast, VCS biomass and hydro power projects perform better than VCS wind power projects. Furthermore, the CDM host region Asia is beneficial compared to Africa and Latin America. On technology level, we find that increasing project durations increase the projects' success except for VCS hydro power projects. Small scale projects perform better compared to large scale projects in the case of VCS wind power projects. Especially, in the cases of CDM and VCS wind and CDM hydro power projects, we observe economies of scale and time. Location-wise, the host region Africa is disadvantageous compared to Asia in the case of CDM energy efficiency projects, whereas, it is beneficial for CDM hydro power projects, although there are off-setting effects. Our results suggest that Latin America is advantageous compared to Asia for CDM biomass projects, despite leveling effects for CDM energy efficiency and hydro power projects. When it comes to CDM wind power projects, there is no significant difference in the performance concerning the host regions. A similarity of CDM biomass and hydro power projects is the partly significant negative singular influence of the number of countries involved, the large scale methodology and the increasing project duration, whereas the combination of large scale projects with an increasing number of involved countries as well as long project durations with an increased number of involved countries influences the projects' success significantly positively. In the factor combinations of the stepwise regression results, balancing effects are present and need to be kept in mind with regard to the interpretation of the results.

\section{Conclusions and Policy Implications}

There are diverse carbon credit regimes with different scopes, regulatory backgrounds and administrative requirements. As there are new carbon credit mechanisms evolving and existing mechanisms are struggling, we focus on the question of what determinants make a carbon credit project successful. These determinants are important because they affect the effectiveness and efficiency of a wide-spread climate policy measure. Identifying success factors of Clean Development Mechanism (CDM) and Verified Carbon Standard (VCS) projects, as representatives for mandatory and voluntary carbon credit regimes, is the aim of this work. To the best of our knowledge, the comparison of success determining factors of different carbon credit regimes has not appeared in the literature so far. We apply ordinary least squares (OLS) and stepwise regression and analyze the project data standardand technology-wise. In particular, we compare CDM and VCS wind power, energy efficiency, hydro power and biomass projects, respectively. Our technology level results do not indicate economies of scale per se. Furthermore, difficulties originating from the large scale project specification are overcome by time in the cases of CDM and VCS wind power projects as well as CDM hydro power projects. Additionally, our results suggest that CDM energy efficiency projects should preferably not be located in African countries. VCS hydro power projects show no economies of time according to our data. Moreover, the number of countries involved in a project associated with economies of time has a significant and positive influence on CDM hydro power and biomass projects. This is a similarity of 
VCS and CDM biomass projects. The number of participating countries in CDM energy efficiency and wind power projects should be as small as possible. The presented results are broadly in line with intuition and the related literature, although they are not always highly significant statistically. The explanation of the variation of the projects' success is sounder with regard to CDM projects. The lacking explanatory power concerning VCS projects might originate from the data availability and the fact that many VCS projects have shorter crediting periods sometimes equivalent to ramp-up phases, whereupon those projects switch to, for example, the CDM. Hence, these ramp-up phases would bias the evaluation.

Author Contributions: Conceptualization, A.v.A., T.S.D. and R.K.; Methodology, A.v.A. and R.K.; Validation, A.v.A., T.S.D. and R.K.; Formal Analysis, A.v.A. and R.K.; Data Curation, A.v.A. and T.S.D.; Writing-Original Draft Preparation, A.v.A. and R.K.; Writing-Review \& Editing, A.v.A. and R.K.; Project Administration, T.S.D. and R.K.; Funding Acquisition, T.S.D. and R.K.

Funding: This research was funded by Deutscher Akademischer Austauschdienst (DAAD) project number: 57037669.

Conflicts of Interest: The authors declare no conflict of interest. The founding sponsors had no role in the design of the study; in the collection, analyses, or interpretation of data; in the writing of the manuscript, and in the decision to publish the results.

\section{References}

1. United Nations; European Commission; International Monetary Fund; Organisation for Economic Co-operation and Development; World Bank. Handbook of National Accounting: Integrated Environmental and Economic Accounting 2003. Available online: https://unstats.un.org/unsd/envaccounting/seea2003.pdf (accessed on 4 June 2018).

2. European Commission. EU ETS Fact Sheet. 2016. Available online: https://ec.europa.eu/clima/sites/ clima/files/factsheet_ets_en.pdf (accessed on 4 June 2018).

3. World Bank; Ecofys. State and Trends of Carbon Pricing 2018; Technical Report; World Bank: Washington, DC, USA, 2018.

4. World Bank; Ecofys; Vivid Economics. State and Trends of Carbon Pricing 2017; Technical Report; World Bank: Washington, DC, USA, 2017.

5. Hamrick, K. Ahead of the Curve: State of the Voluntary Carbon Markets 2015; Technical Report; Forest Trends' Ecosystem Marketplace: Washington, DC, USA, 2015.

6. Conte, M.N.; Kotchen, M.J. Explaining the Price of Voluntary Carbon Offsets. Clim. Chang. Econ. 2010, 1, 93-111. [CrossRef]

7. Kollmuss, A.; Zink, H.; Polycarp, C. Making Sense of the Voluntary Carbon Market. A Comparison of Carbon Offset Standards; Technical Report; World Wildlife Fund (WWF): Berlin, Germany, 2008.

8. Durth, R. Trading of Emission Certificates for Climate Protection: Using Markets and Private Capital for Development. In Greening the Financial Sector: How to Mainstream Environmental Finance in Developing Countries; Köhn, D., Ed.; Springer: Berlin/Heidelberg, Germany, 2012; pp. 207-213.

9. World Bank. State and Trends of the Carbon Market 2010; Technical Report; World Bank: Washington, DC, USA, 2010.

10. Peters-Stanley, M.; Gonzalez, G. Sharing the Stage: State of the Voluntary Carbon Markets 2014; Technical Report; Forest Trends' Ecosystem Marketplace: Washington, DC, USA, 2014.

11. Hamrick, K. Raising Ambition: State of the Voluntary Carbon Market 2016; Technical Report; Forest Trends' Ecosystem Marketplace: Washington, DC, USA, 2016.

12. World Bank. State and Trends of Carbon Pricing 2016; Technical Report; World Bank: Washington, DC, USA, 2016.

13. Taiyab, N. Exploring the Market for Voluntary Carbon Offsets; Markets for Environmental Services; International Institute for Environment and Development: London, UK, 2006.

14. Peters-Stanley, M.; Yin, D. Maneuvering the Mosaic: State of the Voluntary Carbon Markets 2013; Technical Report; Forest Trends' Ecosystem Marketplace: Washington, DC, USA; Bloomberg New Energy Finance: New York, NY, USA, 2013.

15. Castro, P.; Michaelowa, A. Empirical Analysis of Performance of CDM Projects; Technical Report; Climate Strategies: Zurich, Switzerland, 2008. 
16. Zavodov, K. Renewable energy investment and the Clean Development Mechanism. Energy Policy 2012, 40, 81-89. [CrossRef]

17. Jung, M. Host country attractiveness for CDM non-sink projects. Energy Policy 2006, 34, 2173-2184.10.1016/j.enpol.2005.03.014. [CrossRef]

18. Winkelman, A.G.; Moore, M.R. Explaining the differential distribution of Clean Development Mechanism projects across host countries. Energy Policy 2011, 39, 1132-1143.10.1016/j.enpol.2010.11.036. [CrossRef]

19. Rahman, S.M.; Kirkman, G.A. Costs of certified emission reductions under the Clean Development Mechanism of the Kyoto Protocol. Energy Econ. 2015, 47, 129-141.10.1016/j.eneco.2014.10.020. [CrossRef]

20. UNFCCC. Distribution of Registered Projects by Other Party; UNFCCC: Bonn, Germany, 2017.

21. Wooldridge, J. Introductory Econometrics: A Modern Approach; ISE-International Student Edition; Cengage Learning: Mason, OH, USA, 2008.

22. Collett, D. Modelling Binary Data, 2nd ed.; Chapman \& Hall/CRC Texts in Statistical Science: Boca Raton, FL, USA, 2002.

23. Kutner, M. Applied Linear Statistical Models; McGrwa-Hill International Edition; McGraw-Hill Irwin: New York, NY, USA, 2005.

24. UNFCCC. Glossary: CDM Terms; Version 8; UNFCCC: Bonn, Germany, 2015.

25. Kirkman, G.A.; Seres, S.; Haites, E. Renewable energy: Comparison of CDM and Annex I projects. Energy Policy 2013, 63, 995-1001. [CrossRef]

26. UNFCCC. CDM Project Cycle Procedure; UNFCCC: Bonn, Germany, 2015.

27. Kirkman, G.A.; Seres, S.; Haites, E.; Spalding-Fecher, R. Benefits of the Clean Development Mechanism 2012; Technical Report; UNFCCC: Bonn, Germany, 2012.

28. Sawhney, A.; Rahul, M. Examining the regional pattern of renewable energy CDM power projects in India. Energy Econ. 2014, 42, 240-247. [CrossRef]

29. Dorsman, A.; Simpson, J.; Westerman, W. Energy Economics and Financial Markets; Springer: Berlin/Heidelberg, Germany, 2012.

30. Labatt, S.; White, R. Carbon Finance: The Financial Implications of Climate Change; Wiley Finance: Hoboken, NJ, USA, 2007.

(C) 2018 by the authors. Licensee MDPI, Basel, Switzerland. This article is an open access article distributed under the terms and conditions of the Creative Commons Attribution (CC BY) license (http://creativecommons.org/licenses/by/4.0/). 